To be submitted to the Fifteenth International Conference on the Application of Accelerators in Research and Industry, Denton, TX, November 4-7, 1998.
JAN $? 21999$

OSTI

\title{
Solutions to Defect-Related Problems in Implanted Silicon by Controlled Injection of Vacancies by High-Energy Ion Irradiation
}

\author{
E. G. Roth \\ University of North Texas, Denton, TX and
}

Oak Ridge National Laboratory, Oak Ridge, TN

O. W. Holland

Oak Ridge National Laboratory, Oak Ridge, TN

J. L. Duggan

University of North Texas, Denton, TX \begin{abstract}
"The submitted manuscript has been authored by a contractor of the U.S. Government under
contract No. contract Nocordingly, the U.S. Government retains a nonexclusive, royalty-free license to publish or reproduce the published form of this contribution, or allow others to do so, for U.S. Government purposes."
\end{abstract}

\author{
Prepared by the \\ Oak Ridge National Laboratory \\ Oak Ridge, Tennessee 37831 \\ managed by \\ LOCKHEED MARTIN ENERGY RESEARRCH CORP. \\ for the \\ U.S. DEPARTMENT OF ENERGY \\ under contract DE-AC05-96OR22464
}




\section{DISCLAIMER}

This report was prepared as an account of work sponsored by an agency of the United States Government. Neither the United States Government nor any agency thereof, nor any of their employees, make any warranty, express or implied, or assumes any legal liability or responsibility for the accuracy, completeness, or usefulness of any information, apparatus, product, or process disclosed, or represents that its use would not infringe privately owned rights. Reference herein to any specific commercial product, process, or service by trade name, trademark, manufacturer, or otherwise does not necessarily constitute or imply its endorsement, recommendation, or favoring by the United States Government or any agency thereof. The views and opinions of authors expressed herein do not necessarily state or reflect those of the United States Government or any agency thereof. 


\section{DISCLAIMER}

Portions of this document may be illegible in electronic image products. Images are produced from the best available original document. 


\title{
Solutions to Defect-Related Problems in Implanted Silicon by Controlled Injection of Vacancies by High-Energy Ion Irradiation
}

\author{
E. G. Roth \\ University of North Texas, Physics Department, Denton, TX 76203 and \\ Oak Ridge National Laboratory, Solid State Division, Oak Ridge, TN 37831-6048 \\ O. W. Holland \\ Oak Ridge National Laboratory, Solid State Division, Oak Ridge, TN 37831-6048 \\ J. L. Duggan \\ University of North Texas, Physics Department, Denton, TX 76203
}

\begin{abstract}
Amorphization and a dual implant technique have been used to manipulate residual defects that persist following implantation and post-implant thermal treatments. Residual defects can often be attributed to ion-induced defect excesses. A defect is considered to be excess when it occurs in a localized region at a concentration greater than its complement. Sources of excess defects include spatially separated Frenkel pairs, excess interstitials resulting from the implanted atoms, and sputtering. Preamorphizing prior to dopant implantation has been proposed to eliminate dopant broadening due to ion channeling as well as dopant diffusion during subsequent annealing. However, transient-enhanced diffusion (TED) of implanted boron has been observed in pre-amorphized Si. The defects driving this enhanced boron diffusion are thought to be the extended interstitial-type defects that form below the amorphous-crystalline interface during implantation. A dual implantation process was applied in an attempt to reduce or eliminate this interfacial defect band. High-energy, ion implantation is known to inject a vacancy excess in this region. Vacancies were implanted at a concentration coincident with the excess interstitials below the a-c interface to promote recombination between the two defect species. Preliminary results indicate that a critical fluence, i.e., a sufficient vacancy concentration, will eliminate the interstitial defects. The effect of the reduction or elimination of these interfacial defects upon TED of boron will be discussed. Rutherford backscattering/channeling and cross section transmission electron microscopy analyses were used to characterize the defect structure within the implanted layer. Secondary ion mass spectrometry was used to profile the dopant distributions.
\end{abstract}

\section{INTRODUCTION}

Amorphizing the lattice prior to dopant implantation has been suggested as a way to inhibit transient-enhanced diffusion (TED) of B arising from ioninduced defects. It was thought that subsequent solidphase-epitaxial growth (SPEG) of the amorphous layer would restore the lattice to a single-crystal and remove all ion-induced defects, which might drive the transiency. However, even when the B distribution was completely contained within an amorphous layer, enhanced diffusion still occurred, regardless of the amorphizing ion. ${ }^{1-3}$ This enhancement in the diffusivity of $B$ has been attributed to two possible sources of residual defects. The first is the interstitial defects left below the amorphous-crystalline $(a-c)$ interface following SPEG ${ }^{3,4}$ In fact, the dissociation of these defects during annealing has been directly correlated with the enhancement in the diffusivity of $B{ }^{5}$ A second mechanism suggested that lattice strain from the implant damage and, in some cases, trapped defects remained following SPEG of the amorphous layer and were responsible for enhancing dopant diffusion in preamorphized layers. ${ }^{6-9}$ However, a recent experiment involving B implanted within a buried amorphous layer in silicon-on-insulator material confirmed that the interstitial defects below the a-c interface were responsible for TED of $\mathrm{B}$ implanted within amorphous $\mathrm{Si}$, not an intrinsic property of the regrown layer. ${ }^{10}$ In this work, an implantation method is discussed for manipulating such defects as the end-of-range (EOR) damage. The method involves synergistic effects arising from co-implantation of two different ion beams which if applied judiciously, can be used to tailor the distribution of implant damage.

Understanding defect interactions is vital in developing novel approaches to manipulate ion-induced defects. This engineering of the defects to alter their final distribution and morphology is becoming more effective in successfully reducing or removing defect excesses. As a 
result, the formation of extended defects that are stable up to high temperatures can be prevented, thus reducing the total thermal budget needed to eliminate residual implant damage. Several studies have reported on defect interactions that occur during ion irradiation, especially under unique conditions such as high-energy ion implantation. ${ }^{11-15}$ Many of these studies involved implants performed at ambient and liquid nitrogen temperatures. Consequently, much is yet to be learned from implants performed at elevated temperatures. ${ }^{16}$ Actually, synergistic effects involving co-implantation of two different energetic ion beams have been demonstrated ${ }^{16}$ but need to be better understood before this ion technology can be fully utilized. The synergism between dual implants of high- and medium-energy ions is explored; namely the interaction of complementary "excess" defect regimes (discussed below) that follow the implant process for the efficient removal of unwanted defect morphologies.

Following implantation, there are regions within the lattice where a preponderance of one point defect (i.e., vacancy or interstitial) is found over its complement. There are three sources of such defect excesses: spatial separation of Frenkel pairs (FP), the interstitials introduced into the lattice by the added implanted atoms (the plus-one, +1 , state ${ }^{17}$ ), and sputtering (SP) of the surface. Spatial separation of FP arises due to the momentum transfer from the incident ion to the recoil atom, depositing the knockons deeper into the lattice than their vacancy counterparts. ${ }^{18}$ The distribution of vacancies is shallower than the interstitial population and extends to the surface. The inability of the lattice to incorporate implanted ions directly onto lattice sites without defect formation yields the +1 state, a distribution of excess interstitials that mimics the implant profile in both concentration and distribution. Finally, SP of the surface during irradiation enriches the top few monolayers with vacancies. High-energy ion implantation is useful for defect studies because the excess defect populations described above have sufficient spatial separation to survive recombination. This is illustrated in the TRIM (TRansport of Ions in Matter) simulation, in Figure 1, which illustrates the excess defect distributions for $1.25 \mathrm{MeV}, 5 \times 10^{16} / \mathrm{cm}^{2} \mathrm{Si}^{+}$-ions implanted into $\mathrm{Si}^{19}$ This simulation shows the distribution of ion-induced displacements but with the correlated Frenkel pairs removed so that only the excess defect populations remain. This is done by simply subtracting the vacancy distribution from the interstitials and plotting the difference. Physically this is thought to occur dynamically by recombination either during implantation or post-implant annealing. This yields a near-surface concentration of vacancies and an interstitial excess near the ion range, $R_{\mathrm{p}}$. The +1 represents the distribution of implanted ions. A full understanding of the formation and survival of excess defect populations enhances the ability to manipulate these defects in such a way as to increase the effectiveness in gettering applications $^{20-21}$, reducing secondary defect formation ${ }^{22-23}$, or eliminating deleterious effects such as diffusion enhancement.

\section{EXPERIMENTAL}

\section{Below Amorphization Threshold}

Dual implant processes were analyzed to study synergistic effects and to identify possible mechanisms for the manipulation of the excess defect profiles. CzochralskiSi (100) material (p-type, resistivity: $1-10 \Omega \mathrm{cm}$ ) was implanted with $5 \times 10^{16} / \mathrm{cm}^{2}, 1.25 \mathrm{MeV} \mathrm{Si}^{+}$-ions at $250^{\circ} \mathrm{C}$. Following $\mathrm{MeV}$ implantation, $200 \mathrm{keV} \mathrm{Si}{ }^{+}$-ions were implanted with fluences ranging from $10^{15}$ to $10^{16} / \mathrm{cm}^{2}$. The implant temperature of both implants was elevated to $250^{\circ} \mathrm{C}$ to promote local recombination during implantation and prevent amorphization of the lattice. The $\mathrm{MeV}$ implants were performed on a 1.7 MV General Ionex tandem accelerator. The use of a tandem accelerator to implant 28 $\mathrm{amu} \mathrm{Si}{ }^{+}$-ions ensures the beam will be free of contaminants with the same mass-to-charge ratio that are potentially present when using a single-ended machine. In addition, implants were done with a raster-scanned beam to insure uniform implanted areas. Medium-energy implants were performed on a single-ended, mass-analyzed implanter. For these medium-energy implants both above and below the amorphization threshold, the use of a $30 \mathrm{amu} \mathrm{Si}^{+}$-ion beam minimized the probability of beam contamination, especially from $\mathrm{N}_{2}^{+}$or $\mathrm{CO}^{+}$. Si was chosen for these studies of implant damage so the interaction of defects would not be convoluted with chemical effects. Rutherford backscattering (RBS)/channeling analysis with $2.3 \mathrm{MeV} \mathrm{He}{ }^{++}$-ions was the primary tool used to investigate resulting implant damage. A standard surface barrier detector collected backscattered ions at $160^{\circ}$.

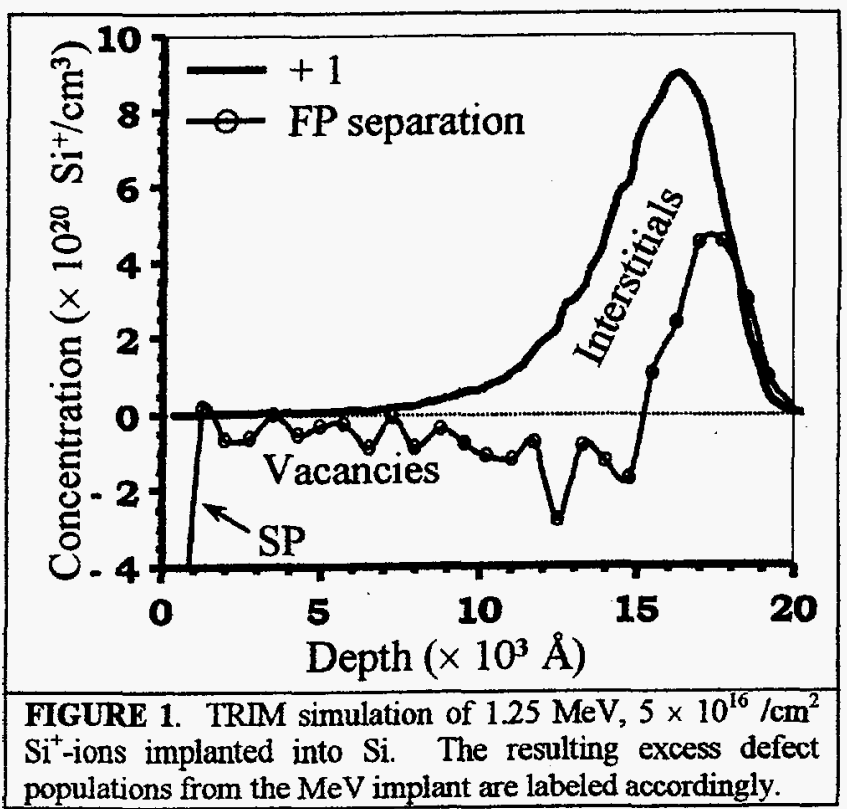




\section{Above Amorphization Threshold}

Utilizing a similar dual implant process, amorphization was used concomitantly with $\mathrm{MeV}$ implantation to remove the defects responsible for $B$ diffusion in pre-amorphized layers. Czochralski-Si (100) samples were pre-damaged with 5 and $6 \times 10^{16} / \mathrm{cm}^{2}, 1.25$ $\mathrm{MeV} \mathrm{Si}^{+}$-ions at $100^{\circ} \mathrm{C}$ and subsequently amorphized with $10^{15} / \mathrm{cm}^{2} \mathrm{Si}^{+}$-ions implanted at both 70 and $140 \mathrm{keV}$ at room temperature (RT). Previous studies showed that when B was implanted into an amorphous layer created with the same amorphizing conditions, significant enhanced diffusion resulted. ${ }^{3}$ This TED resulted following the release of the excess interstitials on the crystalline side of the a-c interface. In this experiment, vacancy-type defects were injected below the a-c interface using high-energy ions to enhance recombination with the interstitial source responsible for TED of B within an amorphous Si layer. Similarly, 40 $\mathrm{keV}, 10^{14} / \mathrm{cm}^{2} \mathrm{~B}^{+}$-ions were implanted at RT within the amorphous layer of the dual implanted material as a probe to determine the effectiveness of the dual implant process on eliminating the interstitial defect band below the a-c interface. Secondary ion mass spectrometry (SIMS) profiled the dopant distributions within the damaged layers, following anneals performed in a conventional quartz-tube furnace under a flowing $\mathrm{Ar}-\mathrm{H}$ (96-4\%) ambient.

\section{RESULTS AND DISCUSSION}

\section{Synergism of Damage from High- and Medium-Energy Si ${ }^{+}$-Ion Implantation}

Damage resulting from co-implantation of highand medium-energetic $\mathrm{Si}^{+}$-ions was studied to understand the synergistic effects that arise during high-temperature irradiation and their dependence on the order of the implantation. These effects will illuminate interesting aspects of the defect interactions, which underlie this method of defect engineering. That is, these results will clarify the role of the excess defect regions left by an initial high-energy implant, namely the excess vacancy region, in eliminating ion-induced interstitials from implants of lower energy $\mathrm{Si}^{+}$-ions. The parameters of the self-ion $\mathrm{MeV}$ implant were fixed $(1.25 \mathrm{MeV}$, $\left.5 \times 10^{16} / \mathrm{cm}^{2} \mathrm{Si}^{+}, 250^{\circ} \mathrm{C}\right)$. The medium-energy $(200 \mathrm{keV})$ $\mathrm{Si}^{+}$-ions were implanted to varying fluences in the range of $10^{15}$ to $10^{16} / \mathrm{cm}^{2}$. The order of these implants was altered as well so the reversibility of the defect interactions could be studied. The implant histories are summarized in Table 1 and will be referred to as implants of Order 1 or Order 2 depending on the order of the highand medium-energy implants.

The total damage resulting from the implants was extracted from RBS/channeling spectra utilizing a routine that extracts ion-implanted damage by subtracting

\begin{tabular}{|c|c|}
\hline TABLE 1. Summary of Order and Parameter of Dual Implants \\
\hline Order & Parameters \\
\hline Order 1 & $\mathrm{MeV}^{2}+\mathrm{Si}^{+}\left(200 \mathrm{keV}, 10^{15}-10^{16} / \mathrm{cm}^{2}, 250^{\circ} \mathrm{C}\right)$ \\
\hline Order 2 & $\mathrm{Si}^{+}\left(200 \mathrm{keV}, 10^{15}-10^{16} / \mathrm{cm}^{2}, 250^{\circ} \mathrm{C}\right)+\mathrm{MeV}^{\mathrm{a}}$ \\
\hline
\end{tabular}

MEV: $\mathrm{Si}^{+}\left(1.25 \mathrm{MeV}, 5 \times 10^{16} / \mathrm{cm}^{2}, 250^{\circ} \mathrm{C}\right)$

a calculated, dechanneled fraction from the original RBS profile. ${ }^{24}$ The dechanneled profile is shown in Figure $2 \mathrm{a}$ as the background trace $(O)$, along with the RBS profile of the implanted Si (-). The resulting damage profile (hatchured area in Fig. 2a), plotted in Figure $2 b$ for damage concentration versus depth, was integrated yielding an areal density of damage. This implant damage for each fluence of $200 \mathrm{keV} \mathrm{Si}{ }^{+}$-ions ranging from $10^{15}$ to $10^{16} / \mathrm{cm}^{2}$ implanted either prior or subsequent to $1.25 \mathrm{MeV}, 5 \times 10^{16} / \mathrm{cm}^{2} \mathrm{Si}^{+}$ions is summarized in Figure 3. The third curve $(A)$ in Figure 3 is the control. This curve gives the sum of the damage for $200 \mathrm{keV} \mathrm{Si}^{+}$-ions and $1.25 \mathrm{MeV} \mathrm{Si}^{+}$-ions when the implants are done separately. Therefore, the control curve reflects the total implanted damage from the respective implants without any synergistic effects. The damage obtained for the dual implants of Order 1 and 2 is compared to the control.

The damage from implants of Order 1 at different fluences of $200 \mathrm{keV} \mathrm{Si}{ }^{+}$-ions increases for increasing fluence as seen previously. ${ }^{14}$ In the control, the damage is higher than that seen in the curve of Order limplants over the entire range of implanted fluence of $200 \mathrm{keV} \mathrm{Si}{ }^{+}$-ions. In fact, the predominant difference is that the damage in the control

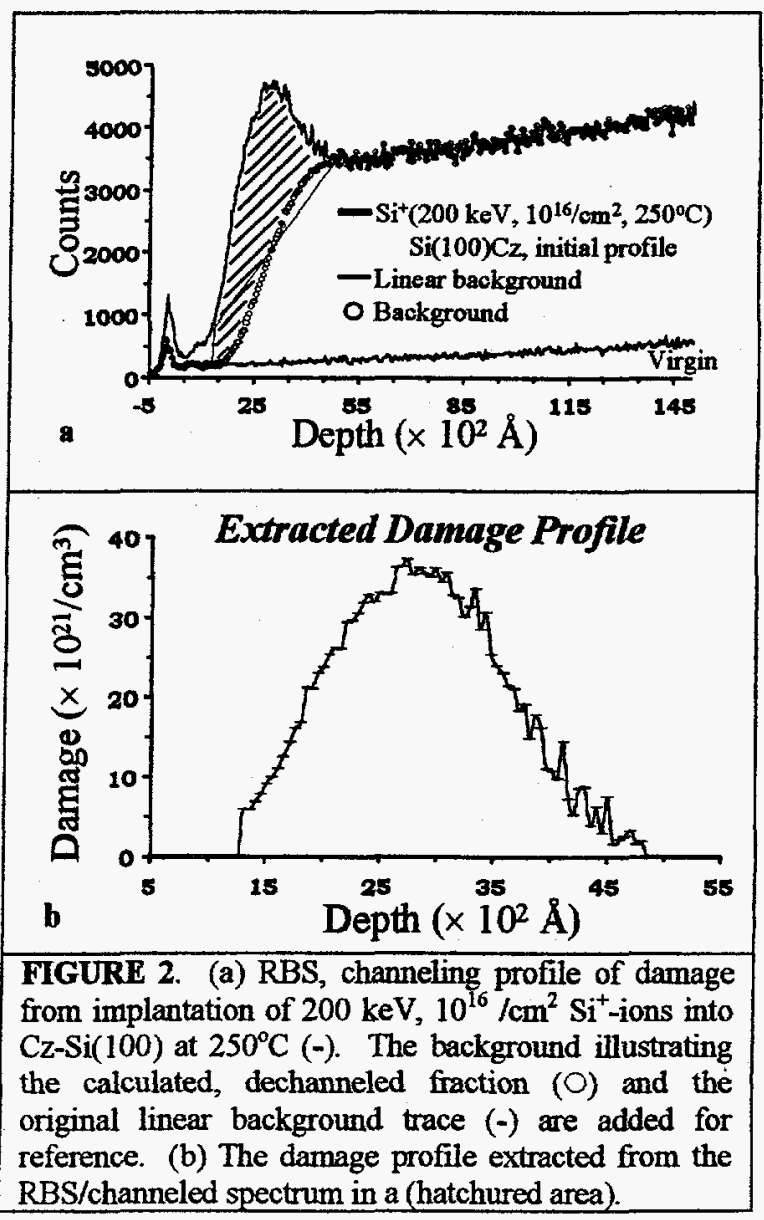




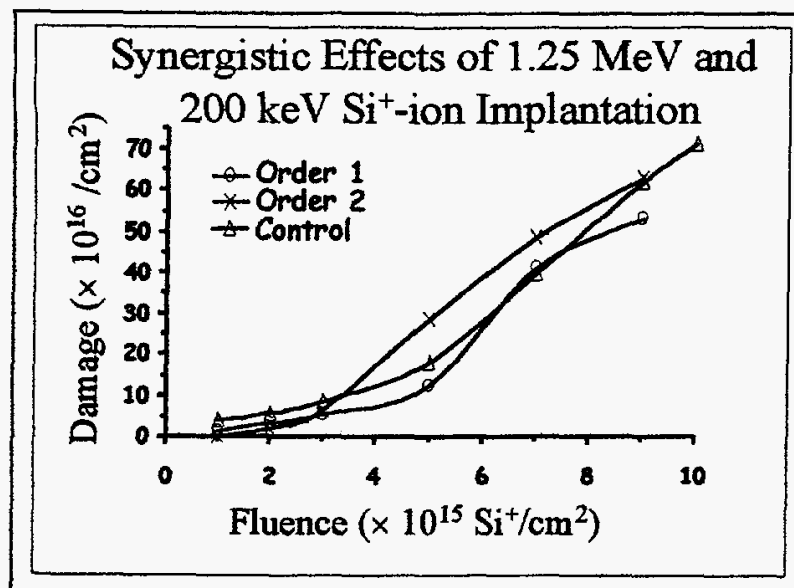

FIGURE 3. Damage extracted from RBS spectra plotted versus implant fluence of the $200 \mathrm{keV} \mathrm{Si}{ }^{+}$-ions. Order 1 reflects damage following implantation of $1.25 \mathrm{MeV}+200$ $\mathrm{keV} \mathrm{Si}{ }^{+}$-ions, and Order 2 shows damage implants are reversed (200 keV + $1.25 \mathrm{MeV} \mathrm{Si}^{+}$-ions). The control contains the sum of the damage from individual implants of $200 \mathrm{keV}$ and $1.25 \mathrm{MeV} \mathrm{Si}^{+}$-ions, i.e., no synergism.

appears offset from the damage of Order 1 implants by a constant amount. This is consistent with the following interpretation of the synergistic effect. The $\mathrm{MeV}$ implant creates an excess vacancy population that peaks around $1 / 2$ $\mathrm{R}_{\mathrm{p}}$ of the $\mathrm{MeV}$ implant. Subsequently implanting 200 $\mathrm{keV} \mathrm{Si}{ }^{+}$-ions fills the holes left by the $\mathrm{MeV}$ implant, thus, the existing vacancy-rich region anneals much of the damage introduced by the $200 \mathrm{keV}$ implant. This filling, in effect, restores the lattice to a defect free state locally, and once the holes are filled, the damage from the 200 $\mathrm{keV}$ implant starts to accumulate within the lattice. Therefore, with a vacancy supersaturation in place from an initial implant of $1.25 \mathrm{MeV} \mathrm{Si}^{+}$-ions, an implant of lower energy $\mathrm{Si}^{+}$-ions promotes the interaction, i.e., recombination, of the vacancies with the interstitials (predominantly +1 state) of the lower-energy self-ions. The initial $\mathrm{MeV}$ implant remained unchanged, hence a constant offset of a few $10^{16} / \mathrm{cm}^{2}$ is observed. TRIM simulations show that this dose roughly corresponds to the condition where the peak of the interstitial excess (from the medium-energy implant) is equivalent to the excess vacancy concentration at $1 / 2 R_{p}$ (produced by $\mathrm{MeV}$ ions).

The synergistic behavior that was observed for the previous dual implants of Order 1 was not the same when the onder of implantation was reversed. Note that the damage from implants of Order 1 were about the same or slightly higher than the damage from implants of Order 2 for the lower fluences, 1 and $2 \times 10^{15} / \mathrm{cm}^{2}$, of $200 \mathrm{keV}$ $\mathrm{Si}^{+}$-ions. Consequently, at the lower fluences of the medium-energy implant, similar behavior for either order of implantation prevailed and recombination between the ion-induced interstitials from the $\mathrm{keV}$ implant and the vacancies from the high-energy implant persisted, perhaps more effectively for implants of Order 2 as the amount of damage was lower. However, just prior to the implant of $3 \times 10^{15} / \mathrm{cm}^{2}, 200 \mathrm{keV} \mathrm{Si}{ }^{+}$-ions, the damage from implants of Order 2 begins to exceed the damage from implants of Order 1, but is still lower than the control. The magnitude of damage for either order of implantation increased for increasing implant fluence of the $200 \mathrm{keV} \mathrm{Si}$ implants. Once past the junction at $3 \times 10^{15} / \mathrm{cm}^{2}, 200 \mathrm{keV}$ $\mathrm{Si}^{+}$-ions, the damage from implants of Order 2 rose abruptly. In fact, the damage from Order 2 type implants was greater than that seen in the control following a critical implant fluence of $4 \times 10^{15} / \mathrm{cm}^{2}, 200 \mathrm{keV} \mathrm{Si}{ }^{+}$-ions. Clearly, the nature of the defect interactions changed from that seen in the implants of Order 1. It appears that the interstitials from the $\mathrm{keV}$ implants are no longer inclined to recombine with the vacancies from the subsequent $\mathrm{MeV}$ implants. Instead, the self-clustering defects appear to dominate possibly beginning at nucleation sites formed following the first implant of $\mathbf{2 0 0}$ $\mathrm{keV} \mathrm{Si}{ }^{+}$-ions. Clearly, the defect interactions responsible for these results are not reversible and are very order dependent. It is clear that for the injected vacancy concentration used in this study, the recombination reaction always dominated during the Order 1 type implants. However, in Order 2 type implants the situation was more complex. It would appear that for a lower fluence of the medium-energy ions, the excess interstitials remained in small complexes, which could be removed by subsequent injection of interstitials. However, at higher doses, these interstitials form more stable configurations (i.e., dislocation loops) that have a stronger affinity to resist recombination.

Therefore, while these studies show some complicated effects, it is clear that, independent or ordering, $\mathrm{MeV}$ ions can be used to inject vacancies over an extended depth to act as recombination sites for interstitials. This is limited in Order 2 type implants to a critical concentration of interstitiais. Following the implant of this critical fluence of $200 \mathrm{keV}$ ions, nucleation sites are formed which may be origins for self-clustering to occur, thereby yielding damage growth.

\section{Removal of Crystalline Defects Below a-c Interface}

The above study reveals a dual implant process that can be used to substantially modify as-implanted profiles. This dual implant process was adapted to a situation where the surface layer of a Si lattice, pre-damaged with $1.25 \mathrm{MeV}$ $\mathrm{Si}^{+}$-ions, was amorphized The synergism of co-implants was exploited in an attempt to remove the interstitial defects found below the a-c interface, known to drive TED of B implanted within an amorphous layer. $\mathrm{MeV}$ ions inject a high concentration of vacancies in this region to induce recombination. As such, the $\mathrm{MeV}$ ions are used as a tool to artificially promote the recombination of excess defects within the lattice. Reiterating, this is illustrated in the above experiment where damage annealing was observed (Fig. 3, $(\theta)$ curve) following implantation of $200 \mathrm{keV} \mathrm{Si}^{+}$-ions into a lattice initially implanted with $1.25 \mathrm{MeV} \mathrm{Si}^{+}$-ions.

Damage in the as-implanted sample and in samples annealed at $800^{\circ} \mathrm{C}$ for 20 minutes were characterized with 
RBS/channeling (Fig. 4). A randomly aligned spectrum of a virgin or unimplanted lattice was added for reference. The initial profile ( $\square$ ) indicates the presence of an amorphous layer that extends from approximately $2500 \AA$ within the lattice continuously to the surface. The amorphous layer is certainly continuous to the surface because the backscattered yield is the same as that from the randomly aligned sample spanning the width of the layer to the surface. Also, a channeled profile of a virgin lattice was utilized as a reference in Figure 4, so that any rise in the backscattered yield above that observed in the virgin spectrum would indicate lattice damage resulting from ion implantation processing. The sample that was amorphized only, i.e., saw no prior implant of $\mathrm{MeV}$ ions, was regrown with SPEG at $800^{\circ} \mathrm{C}$ for 20 minutes and used as a control to determine the effectiveness of $\mathrm{MeV}$ implantation in eliminating the interstitial defect band below the a-c interface. In the control spectrum $(x)$, following annealing, the amorphous layer was completely regrown and a defect band was residual below the original a-c interface. The goal of this experiment was to eliminate this EOR defect band via the selective injection of vacancies by high-energy implantation prior to amorphization.

The material was first pre-damaged with $5 \times 10^{16} / \mathrm{cm}^{2}, 1.25 \mathrm{MeV} \mathrm{Si}^{+}$-ions at $100^{\circ} \mathrm{C}$ which corresponds to vacancy concentration of about $1 \times 10^{20} / \mathrm{cm}^{3}$ (determined from TRIM ${ }^{19}$ ). The $\mathrm{MeV}$ implanted sample was amorphized and annealed at $800^{\circ} \mathrm{C}$ for 20 minutes. In Figure 4 , the profile of the $5 \times 10^{16} / \mathrm{cm}^{2}$ implanted sample $(\sharp)$, following annealing, exhibits regrowth of the amorphous layer along with a defect band which remains, although significantly reduced from that seen in the control sample, below the ac interface. So, an implant of $5 \times 10^{16} / \mathrm{cm}^{2}, 1.25 \mathrm{MeV}$

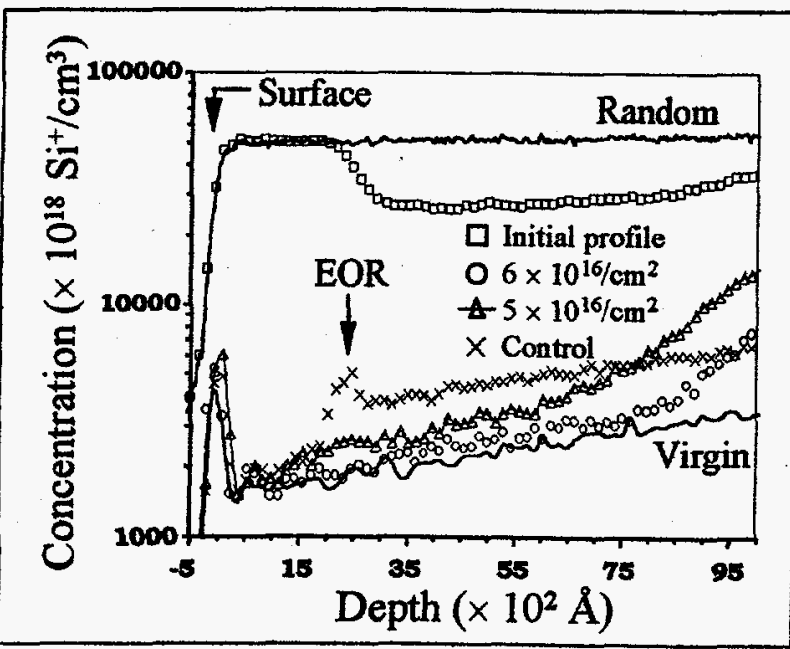

FIGURE 4. Channeled spectra of Cz-Si(100) implanted with 5 and $6 \times 10^{16} / \mathrm{cm}^{2}, 1.25 \mathrm{MeV} \mathrm{Si}^{+}$-ions at $250^{\circ} \mathrm{C}$, amorphized with 70 and $140 \mathrm{keV}, 10^{15} / \mathrm{cm}^{2} \mathrm{Si}^{+}$-ions at $\mathrm{RT}$, and regrown at $800^{\circ} \mathrm{C}$ for 20 minutes. The dual implants were compared to a control that was amorphized only and annealed under the same conditions. The initial, randomly oriented, and virgin profiles were added for reference.
$\mathrm{Si}^{+}$-ions did not produce a vacancy supersaturation sufficient to completely eliminate the excess interstitials below the a-c interface. However, increasing the implant fluence of 1.25 $\mathrm{MeV} \mathrm{Si}{ }^{+}$-ions $20 \%$ to $6 \times 10^{16} / \mathrm{cm}^{2}$ produced a more favorable result. The backscattered yield from the sample implanted with $1.25 \mathrm{MeV}, 6 \times 10^{16} \mathrm{~cm}^{2} \mathrm{Si}^{+}$-ions, amorphized, and regrown was consistent with the virgin (or unimplanted) profile (Fig. 4). Thus, with the sensitivity of the RBS/channeling technique, the excess defects residual below the a-c interface were successfully eliminated with the excess vacancies from high-energy implantation of $6 \times 10^{16}$ $/ \mathrm{cm}^{2} \mathrm{Si}^{+}$-ions. Pictorial evidence obtained with cross-section transmission electron microscopy (XTEM), published elsewhere, verifies the above RBS/channeling data, such that the initial implant of $1.25 \mathrm{MeV}, 6 \times 10^{16} / \mathrm{cm}^{2} \mathrm{Si}^{+}$-ions prior to amorphization completely eliminated the defect band typically found below the a-c interface. ${ }^{25}$ So, the synergistic effect between an initial $\mathrm{MeV}$ implant and subsequent implantation of lower-energy $\mathrm{Si}^{+}$-ions of damage annealing was reproduced for a sample implanted with $\mathrm{MeV}$ ions and then amorphized. The defect band typically found below the a-c interface for a sample amorphized only (the control, $(x)$ ) was eliminated with a MeV implant of $6 \times 10^{16} / \mathrm{cm}^{2} \mathrm{Si}^{+}$-ions prior to amorphization.

A B distribution was intended as a probe to again test the effectiveness of the dual implant process in eliminating the interstitial defects residual below the a-c interface. B was implanted within the amorphous layer of a sample pre-damaged with $1.25 \mathrm{MeV}, 6 \times 10^{16} / \mathrm{cm}^{2} \mathrm{Si}^{+}$-ions at $100^{\circ} \mathrm{C}$. SIMS profiles of $B$ both before and after annealing are shown in Figure 5. When compared to the initial profile, it is obvious that the $\mathrm{B}$ distribution within the amorphous layer of the dual implanted sample $(O)$ underwent little or no movement following the diffusion anneal at $800^{\circ} \mathrm{C}$ for 20 minutes. This is compared to $B$ implanted into a sample that was amorphized only $(x)$, where significant TED was observed following annealing at $800^{\circ} \mathrm{C}$ for 15 minutes. This is consistent with the elimination of the interstitial defects below the a-c interface and thus corroborates the physical evidence provided by TEM and RBS.

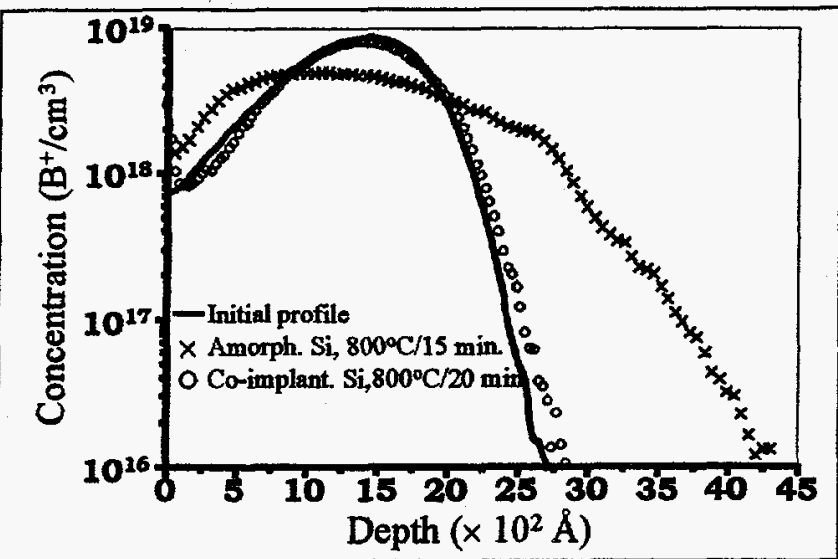

FIGURE 5. SMS profiles of $40 \mathrm{keV}, 10^{14} / \mathrm{cm} 2 \mathrm{~B}^{+}$implanted into amorphous $\mathrm{Si}$ at $\mathrm{RT}$, annealed at $800^{\circ} \mathrm{C}$ for 15 minutes, and amorphous Si pre-damaged with $1.25 \mathrm{MeV}, 6 \times 10^{16} / \mathrm{cm}^{2}$ $\mathrm{Si}^{+}$-ions at $100^{\circ} \mathrm{C}$, annealed at $800^{\circ} \mathrm{C}$ for 20 minutes. The initial profile is added for reference. 


\section{CONCLUSION}

The characterization of damage resulting from dual implants of $\mathrm{Si}^{+}$-ions at different energies revealed a synergy between the two energetic implants dependent upon the order of implantation. Following implantation of high-energy $\mathrm{Si}^{+}$-ions with an implant of mediumenergy $(200 \mathrm{keV})$ ions results in damage annealing, i.e., the 'holes' of the vacancy supersaturated region left by the high-energy implant were filled with the excess interstitials $(+1)$ from a subsequent $\mathrm{keV}$ implant. Once the holes were filled, damage from the implant of 200 $\mathrm{keV} \mathrm{Si}{ }^{+}$-ions began to grow, but, ultimately, it remained less than the damage residual in a sample implanted with $200 \mathrm{keV} \mathrm{Si}{ }^{+}$-ions only. However, the synergism is different for implants of reversed order, $\mathrm{keV}$ ions implanted prior to $\mathrm{MeV}$ ions, once a critical concentration of interstitials is surpassed. The lower fluences of 200 $\mathrm{keV}$ ions, 1 and $2 \times 10^{15} / \mathrm{cm}^{2}$, revealed annealing characteristics similar to that found for dual implants of $\mathrm{MeV}+\mathrm{keV} \mathrm{Si}{ }^{+}$-ions (Order 1). However, as the implant fluence of the $200 \mathrm{keV}$ implant was increased, an abrupt rise in the damage occurred beginning at an implant fluence of $5 \times 10^{15} / \mathrm{cm}^{2}, 200 \mathrm{keV} \mathrm{Si}{ }^{+}$-ions. Obviously, the defects formed more stable configurations that resist recombination. Possible origins of these self-clustering are the nucleation sites resulting from the initial $\mathrm{keV}$ implant. Further study of these defect interactions will illuminate the full effect, damage growth or annealing, of subsequent implantation on existing defect structures within the implanted volume.

Applying this synergism between initial highenergy implants and subsequent medium-energy implants, a novel implant process was developed that eliminated the defect band typically found following regrowth of an amorphous layer continuous to the surface. Vacancies injected into the near-surface layer by $\mathrm{MeV}$ implantation were used to artificially promote the recombination with interstitial defects residual below the original a-c interface following SPEG. From RBS/channeling, it was evident that implanting a sufficient fluence of $1.25 \mathrm{MeV}$, $6 \times 10^{16} / \mathrm{cm}^{2} \mathrm{Si}^{+}$-ions at $100^{\circ} \mathrm{C}$, i.e., injecting a sufficient vacancy concentration, eliminated the interstitial defect band below the interface of the SPEG layer. The removal of this defect band was confirmed with SIMS analysis of $B$ implanted within the amorphous layer of the dual implanted sample. No movement of the $B$ distribution was observed following a diffusion anneal at $800^{\circ} \mathrm{C}$ for 20 minutes. This confirmed the absence of any source of interstitial defects left below the a-c interface to drive enhanced diffusion following a thermal process.

\section{ACKNOWLEDGENTS}

Research was sponsored by the Division of Materials Science, U.S. Department of Energy under Contract DE-AC05-96OR22464 with Lockheed Martin
Energy Research Corporation. This work was also supported in part by a grant from the Robert A. Welch Foundation.

\section{REFERENCES}

1. Peterström, S. and Svensson, B. G., J. Appl. Phys., 71(3), 1215-1218 (1992).

2. Kim, Y. M, Lo, G. Q., Kwong, D. L., Tseng, H. H., and Hance, R., Appl. Phys. Lett., 55(22), 2316-2318 (1989).

3. Roth, E. G., Holland, O. W., Venezia, V. C., and Nielsen, Bent, J. Electon. Mater., 26(11), 1349-1354 (1997).

4. Claverie, Alain, Laânab, Larbi, Bonafos, Caroline, Bergaud, Christian, Martinez, Augustin, and Mathiot, Daniel, Nucl. Instr. and Meth., B 96, 202-209 (1995).

5. Bonafos, C., Omri, M., de Mauduit, B., BenAssayag, G., Claverie, A., Alquier, D., Martinez, A., and Mathiot, D., $J$. Appl, Phys., 82(6), 2855-2861 (1997).

6. Pennycook, S. J., Narayan, J., and Holland, O. W., J. AppL. Phys., 55(4), 837-840 (1984).

7. Pennycook, S. J., Narayan, J., and Holland, O. W., J. Crystal Growth, 70, 597-601 (1984).

8. Pennycook, S. J., Narayan, J., and Holland, O. W., $J$. Electrochem. Soc., 132(8), 1962-1968 (1985).

9. Solmi, S., Angelucci, R., Cembali, F., Servidori, M. and Anderle, M., Appl. Phys. Lett., 51(5), 331-333 (1987).

10. Roth, E. G., Holland, O. W., and Thomas, D. K., Appl. Phys. Lett., submitted for publication.

11. Holland, O. W., El-Ghor, M. K, and White, C. W., Appl. Phys. Lett., 53(14), 1282-1284 (1988).

12. Holland, O. W., El-Ghor, M. K. and White, C. W., Mat. Res. Soc. Symp. Proc., Vol. 147, 169-178 (1989).

13. Holland, O. W., White, C. W., El-Ghor, M. K., and Budai, J. D., J. Appl. Phys., 68(5), 2081-2086(1990).

14. Holland, O. W. and White, C. W., Nucl. Instr. and Meth., B59/60, 353-362 (1991).

15. El-Ghor, M. K., Holland, O. W., White, C. W., and Pennycook, S. J., J. Mater. Res., 5(2), 352-359 (1990).

16. Holland, O. W., Nielsen, Bent, and Budai, J. D., Mat. Res. Soc. Symp. Proc., Vol. 396, 15-20 (1996).

17. Giles, M. D., J. Electrochem. Soc., 138, 1160 (1991).

18. Holland, O. W., Xie, L., Nielsen, B., and Zhou, D. S., J. Electron. Mater., 25(1), 99 (1996).

19. Biersack, J. and Haggmark, L., Nucl. Instr. and Meth., 174, 257 (1980).

20. Tamura, M, Ando, T., and Ohyu, K., Nucl. Instr. and Meth., B59/60, 572-583 (1991).

21. Wong, H., Cheung, N. W., Chu, P. K., Liu, J., and Mayer, J. W., Appi. Phys. Lett., 52(12), 1023-1025 (1988).

22. Lu, W. X., Qian, Y. H., Tian, R. H. Wang, Z. L., Schreutelkamp, R. J., Liefting, J. R., and Saris, F. W., Appl. Phys. Lett., 55 (18), 1838-1840 (1989)

23. Schreutelkamp, R. J., Lu, W. X., Liefting, J. R, Raineri, V., Custer, J. S., and Saris, F. W., Nucl. Instr. Meth., B59/60, 614618 (1991).

24. Chu, Wei-Kan, Mayer, James W., and Nicolet, Marc-A., Backscattering Spectrometny, New York: Academic Press, 1978, ch 8, pp. 255-263.

25. Roth, E. G., Holland, O. W., and Meldrum, A., in Silicon Materials Science and Technology 1998 (Proceedings of the Eighth International Symposium on Silicon Materials Science and Technology, Pennington, NJ, 1998), Huff, H. R, Tsuya, H., and Gösele, U., Editors, PV 98-1, 938-947 (1998). 